\title{
CORRELATED ROUGHNESS EFFECTS IN THE GIANT MAGNETORESISTANCE OF MAGNETIC MULTILAYERS
}

\author{
G. Palasantzas ${ }^{a}$, J. Barnaś ${ }^{b}$ And J.Th.M. De Hosson ${ }^{a}$ \\ ${ }^{a}$ Department of Applied Physics, Materials Science Center and Netherlands Institute \\ for Metals Research \\ University of Groningen, Nijenborgh 4, 9747 AG Groningen, The Netherlands \\ ${ }^{b}$ Department of Physics, Adam Mickiewicz University \\ Umultowska 85, 61-614 Poznań, Poland
}

\begin{abstract}
Interface roughness effects on the giant magnetoresistance in magnetic multilayers are analysed theoretically for structures with non-conformal correlated interfaces. The roughness of each interface is described in terms of the $K$-correlation model and is characterized by the roughness exponent $H(0 \leq H<1)$, correlation length $\xi$, and rms roughness amplitude $\Delta$. Coherent scattering by different interfaces is also taken into account.
\end{abstract}

PACS numbers: 72.15.Gd, 73.40.Jn

Electrical resistance of metallic multilayers composed of magnetic films separated by nonmagnetic spacers varies when magnetic moments of the ferromagnetic layers rotate from antiparallel to parallel alignment [1]. This effect, known as giant magnetoresistance (GMR), is described quantitatively by the factor $\left(R_{\mathrm{ap}}-R_{\mathrm{p}}\right) / R_{\mathrm{p}}$, where $R_{\mathrm{p}}$ and $R_{\text {ap }}$ denote the film resistance for the parallel and antiparallel configurations, respectively. The GMR effect can be accounted for by taking into account spin asymmetry in scattering probabilities [2] and/or in electronic band structure. Theoretical treatment of the effect is significantly complicated by electron scattering on interface roughness, and much experimental and theoretical work has been done to explore this problem [3]. Some theoretical descriptions of GMR show that the interface roughness can enhance or reduce GMR generated by spin dependent scattering on structural defects inside the ferromagnetic films [4]. The problem, however, is more complex as experiments indicate that interfaces can have fractality roughness exponents in the whole range from $H=0$ to $H=1$ [5]. This, in turn, have a significant influence on GMR [6]. Up to now theoretical descriptions of GMR were limited to situations where consecutive interfaces were not correlated (no cross correlation) and different interfaces scattered incoherently. However, experiments show that in real multilayers a finite degree of correlation between interfaces (with different roughness parameters $\Delta, \xi$, and $H$ ) can exist. 
We consider two ferromagnetic films of thicknesses $d_{1}$ and $d_{2}$, which are separated by a non-magnetic film of thickness $d_{0}$. For simplicity, we assume here a spin-polarized free-electron-like model to describe the electronic structure of the conduction electrons in the ferromagnetic films. To emphasise the role of interface roughness we also neglect electron scattering on bulk defects (e.g. on impurities). The two interfaces (distinguished in the following with the index $\beta, \beta=1,2$ ) are located at $z=z_{\beta}+h_{\beta}(\boldsymbol{R})$, with $z_{1}=d_{1}$ and $z_{2}=d_{1}+d_{0}$. Here, $h_{\beta}(\boldsymbol{R})$ are random roughness fluctuations (assumed single valued functions of $\boldsymbol{R} \equiv(x, y)$, with $\left.\left\langle h_{\beta}(\boldsymbol{R})\right\rangle=0\right)$. We assume an isotropic auto-correlation, $C_{\beta}(\boldsymbol{R})=\left\langle h_{\beta}(\boldsymbol{R}) h_{\beta}(0)\right\rangle$, and cross-correlation, $C_{12}(\boldsymbol{R})=\left\langle h_{1}(\boldsymbol{R}) h_{2}(0)\right\rangle$ functions. For simplicity, any roughness of the outer surfaces (at $z=0$ and $z=d_{1}+d_{0}+d_{2} \equiv L$, with $L$ denoting the total thickness of the structure) will be neglected.

For a particular magnetization configuration, the global in-plane conductivity $g$ is given by [4]

$$
g=\frac{2 e^{2}}{\hbar L} \sum_{\sigma} \sum_{\mu=1}^{N_{\sigma}} \sum_{\mu^{\prime}=1}^{N_{\sigma}}\left(E_{\mathrm{F}}-\epsilon_{\mu \sigma}\right)\left(E_{\mathrm{F}}-\epsilon_{\mu^{\prime} \sigma}\right)\left[C_{\sigma}^{-1}\left(E_{\mathrm{F}}\right)\right]_{\mu \mu^{\prime}},
$$

where $\left[C_{\sigma}\left(E_{\mathrm{F}}\right)\right]_{\mu \mu^{\prime}}=\left[C_{\sigma}^{\mathrm{in}}\left(E_{\mathrm{F}}\right)\right]_{\mu \mu^{\prime}}+\left[C_{\sigma}^{\mathrm{coh}}\left(E_{\mathrm{F}}\right)\right]_{\mu \mu^{\prime}}, N_{\sigma}$ is the number of occupied $2 d$ minibands for spin $\sigma(\sigma=\uparrow, \downarrow)$ and $F_{\mathrm{F}}$ denotes the Fermi energy. The matrix elements $\left[C_{\sigma}^{\mathrm{in}}\left(E_{\mathrm{F}}\right)\right]_{\mu \mu^{\prime}}$ describe incoherent scattering by the interfaces and are given in Ref. [4]. The matrix elements $\left[C_{\sigma}^{\text {coh }}\left(E_{\mathrm{F}}\right)\right]_{\mu \mu^{\prime}}$, on the other hand, describe coherent scattering by different interfaces. Following [4] and making use of the approximations $\left\langle h_{\beta}^{3}(\boldsymbol{R}) h_{\beta^{\prime}}\left(\boldsymbol{R}^{\prime}\right\rangle \approx \Delta_{\beta}^{2}\left\langle h_{\beta}(\boldsymbol{R}) h_{\beta^{\prime}}\left(\boldsymbol{R}^{\prime}\right)\right\rangle,\left\langle h_{\beta}^{2}(\boldsymbol{R}) h_{\beta^{\prime}}^{2}\left(\boldsymbol{R}^{\prime}\right)\right\rangle \approx \Delta_{\beta}^{2} \Delta_{\beta^{\prime}}^{2}\right.$ and $\left\langle h_{\beta}^{3}(\boldsymbol{R}) h_{\beta^{\prime}}^{3}\left(\boldsymbol{R}^{\prime}\right)\right\rangle \approx \Delta_{\beta}^{2} \Delta_{\beta^{\prime}}^{2}\left\langle h_{\beta}(\boldsymbol{R}) h_{\beta^{\prime}}\left(\boldsymbol{R}^{\prime}\right)\right\rangle$, we obtain

$$
\left[C_{\sigma}^{\mathrm{coh}}\left(E_{\mathrm{F}}\right)\right]_{\mu \mu^{\prime}}=\delta_{\mu \mu^{\prime}} Q_{\mu \sigma}^{2} \sum_{\nu=1}^{N_{\sigma}} P_{\mu \nu \sigma}^{\mathrm{coh}}+Q_{\mu \sigma} Q_{\mu^{\prime} \sigma} T_{\mu \mu^{\prime} \sigma}^{\mathrm{coh}},
$$

where

$$
\begin{aligned}
P_{\mu \nu \sigma}^{\mathrm{coh}} & =-2 U_{1 \sigma} U_{2 \sigma} L_{12 \sigma}^{\mu \nu} \int_{0}^{2 \pi}\left\langle\left|h_{12}\left(Q_{\mu \nu}^{\sigma}\right)\right|^{2}\right\rangle \mathrm{d} \theta \\
T_{\mu \nu \sigma}^{\mathrm{coh}} & =2 U_{1 \sigma} U_{2 \sigma} L_{12 \sigma}^{\mu \nu} \int_{0}^{2 \pi}\left\langle\left|h_{12}\left(Q_{\mu \nu}^{\sigma}\right)\right|^{2}\right\rangle \cos \theta \mathrm{d} \theta \\
L_{12 \sigma}^{\mu \nu} & =\psi_{\mu \sigma}\left(z_{1}\right) \psi_{\nu \sigma}\left(z_{1}\right)\left[\psi_{\mu \sigma}\left(z_{2}\right) \psi_{\nu \sigma}\left(z_{2}\right)+\frac{1}{4} \Delta_{2}^{2} \psi_{\mu \sigma}^{\prime}\left(z_{2}\right) \psi_{\nu \sigma}^{\prime}\left(z_{2}\right)\right] \\
& +\frac{1}{4} \Delta_{1}^{2} \psi_{\mu \sigma}^{\prime}\left(z_{1}\right) \psi_{\nu \sigma}^{\prime}\left(z_{1}\right)\left[\frac{1}{4} \Delta_{2}^{2} \psi_{\mu \sigma}^{\prime}\left(z_{2}\right) \psi_{\nu \sigma}^{\prime}\left(z_{2}\right)+\psi_{\mu \sigma}\left(z_{2}\right) \psi_{\nu \sigma}\left(z_{2}\right)\right]
\end{aligned}
$$

Here, $\psi_{\mu \sigma}(z)$ are the wave functions corresponding to the miniband edges and $\psi_{\mu \sigma}^{\prime}(z)$ are their first derivatives. In Eqs. $(3,4)\left\langle\left|h_{12}\left(Q_{\mu \nu}^{\sigma}\right)\right|^{2}\right\rangle$ is the Fourier transform of the cross-correlation function, $Q_{\mu \nu}^{\sigma}$ is defined as $Q_{\mu \nu}^{\sigma}=\left(Q_{\mu \sigma}^{2}+Q_{\nu \sigma}^{2}-\right.$ $\left.2 Q_{\mu \sigma} Q_{\nu \sigma}\right)^{1 / 2}$, and $U_{\beta \sigma}$ is the spin-dependent potential step at the $\beta$-th interface.

For a self-affine fractal interface, the Fourier transform $\left\langle|h(q)|^{2}\right\rangle$ of $C(r)$ (the interface index $\beta$ has been omitted here and will be restored when necessary) 
has the scaling behavior $\left\langle|h(q)|^{2}\right\rangle \sim q^{-2-2 H}$ if $q \xi \gg 1$, and $\left\langle|h(q)|^{2}\right\rangle \sim$ const if $q \xi \ll 1$ [7]. The exponent $H$ is associated with a local fractal dimension $D=3-H$. This scaling behavior is described by the $K$-correlation model [8]

$$
\left\langle|h(q)|^{2}\right\rangle=\frac{2 \pi \Delta^{2} \xi^{2}}{\left(1+a q^{2} \xi^{2}\right)^{1+H}} .
$$

The normalization condition $\int_{0<q<q_{\mathrm{c}}}\left\langle|h(q)|^{2}\right\rangle \mathrm{d}^{2} q=(2 \pi \Delta)^{2}$ yields the following equation for the parameter $a: a=(1 / 2 H)\left[1-\left(1+a q_{c}{ }^{2} \xi^{2}\right)^{-H}\right]$ for $0<H \leq 1$ and $a=(1 / 2) \ln \left(1+a q_{\mathrm{c}}^{2} \xi^{2}\right)$ for $H=0$. Here $q_{\mathrm{c}}$ is the upper cut-off, $q_{\mathrm{c}}=\pi / a_{0}$, where $a_{0}$ is the inter-atomic spacing. In numerical calculations we assume that $\left\langle\left|h_{12}(q)\right|^{2}\right\rangle=\left[\left\langle\left|h_{1}(q)\right|^{2}\right\rangle\left\langle\left|h_{2}(q)\right|^{2}\right\rangle\right]^{1 / 2}$.
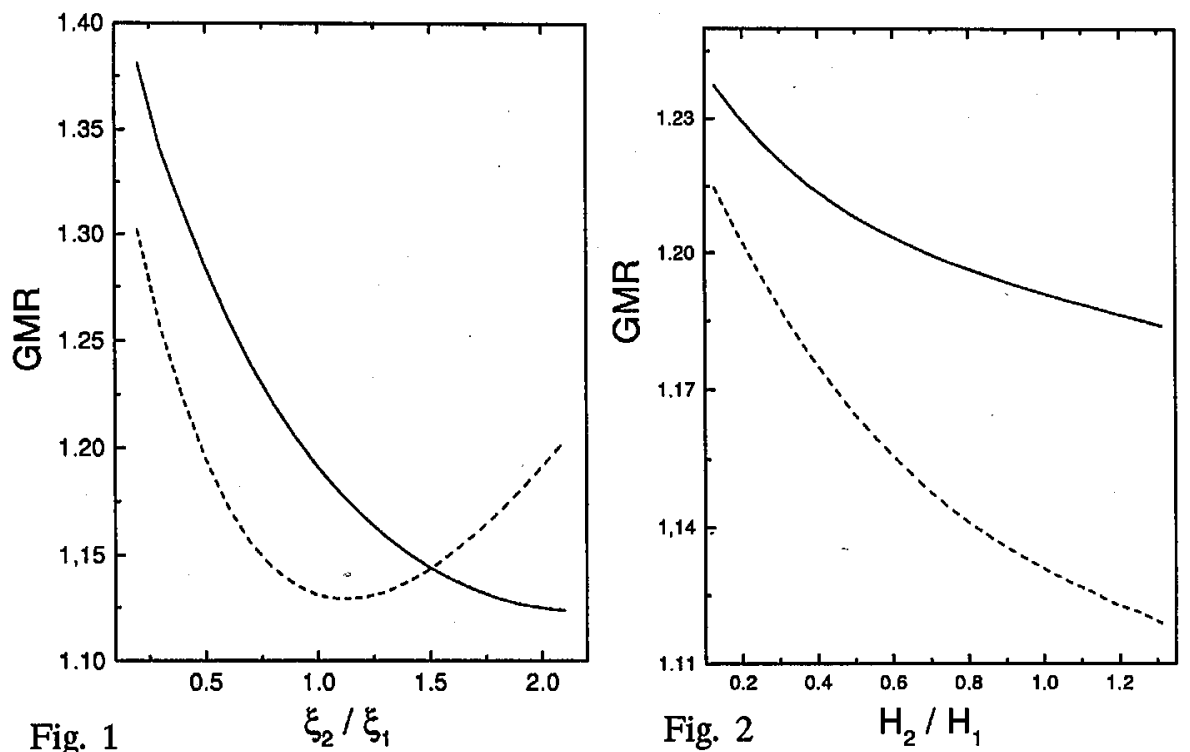

Fig. 1. GMR plotted as a function of $\xi_{2} / \xi_{1}$ (with $\xi_{1}=2 \mathrm{~nm}$ ) for $d_{1}=d_{2}=4 \mathrm{~nm}$, $d_{0}=2 \mathrm{~nm}, E_{\mathrm{F}}=0.3 \mathrm{eV}, a_{0}=0.3 \mathrm{~nm}, \Delta_{1}=\Delta_{2}=0.3 \mathrm{~nm}, H_{1}=H_{2}=0.8$, and for $U_{\beta \sigma}=0.2 \mathrm{eV}$ for spin-minority electrons and $U_{\beta \sigma}=0.1 \mathrm{eV}$ for spin-minority ones. The solid (dashed) line corresponds to the case where the interference effects due to scattering on correlated interfaces are included (ignored).

Fig. 2. GMR plotted as a function of $H_{2} / H_{1}$ for a constant $H_{1}\left(H_{1}=0.8\right)$ and for $\xi_{1}=\xi_{2}=2 \mathrm{~nm}$. The other parameters are the same as in Fig. 1. The solid (dashed) line corresponds to the case where the interference effects due to scattering on correlated interfaces are included (ignored).

Figure 1 shows GMR vs. $\xi_{2} / \xi_{1}$ for a constant $\xi_{1}, \xi_{1}=2 \mathrm{~nm}$. The dashed line corresponds there to the situation where the interference effects due to correlated roughness are ignored, while the solid line corresponds to the case where those effects are included. In Fig. 2, on the other hand, we show GMR vs. $H_{2} / H_{1}$ for a constant $H_{1}, H_{1}=0.8$. From both curves it follows that GMR decreases with 
increasing $H_{2}$ and with increasing $\xi_{2}$. Thus, GMR is very sensitive to correlations between the interface roughness of consecutive interfaces in a multilayer structure.

J.B. acknowledges support through the research project 2 P03B 07514 of the Committee for Scientific Research.

\section{References}

[1] M.N. Baibich, J.M. Broto, A. Fert, F. Nguyen van Dau, F. Petroff, P. Etienne, G. Creuzet, A. Friederich, J. Chazelas, Phys. Rev. Lett. 61, 2472 (1988).

[2] J. Barnaś, A. Fuss, R.E. Camley, P. Grunberg, W. Zinn, Phys. Rev. B 42, 8110 (1990); S. Zhang, P.M. Levy, Phys. Rev. B 43, 11048 (1992).

[3] E.E. Fullerton, D.M. Kelly, J. Guimpel, I.K. Schuller, Y. Bruynseraede, Phys. Rev. Lett. 68, 859 (1992); P. Belien, R. Schad, C.D. Potter, G. Verbanck, V.V. Moshchalkov, Y. Bruynseraede, Phys. Rev. B 50, 9957 (1994).

[4] J. Barnaś, Y. Bruynseraede, Europhys. Lett. 32, 167 (1995).

[5] R. Paniago, R. Forest, P.C. Chow, S.C. Moss, S.S.P. Parkin, D. Cookson, Phys. Rev. B 52, 17052 (1995).

[6] J. Barnaś, G. Palasantzas, J. Appl. Phys. 82, 3950 (1997).

[7] P. Meakin, Phys. Reports 235, 191 (1993).

[8] G. Palasantzas, Phys. Rev. B 48, 14472 (1993); ibid. 49, 5785(E) (1994). 